\title{
The Treatment Outcomes in Children with Medulloblastoma
}

\begin{tabular}{|c|c|c|}
\hline Author(s) & \multicolumn{2}{|c|}{ 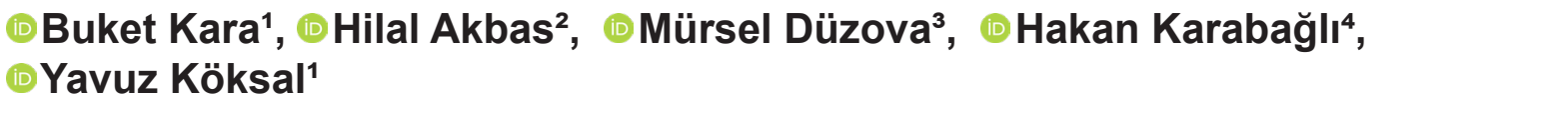 } \\
\hline Affiliation(s) & $\begin{array}{l}{ }^{1} \text { Department of Pediatric Hematology and Oncolog } \\
{ }^{2} \text { Department of Pediatric Hematology and Oncolog } \\
\text { Health Konya City Hospital, Konya, Turkey } \\
{ }^{3} \text { Department of Radiation Oncology, Selcuk Univer } \\
{ }^{4} \text { Department of Neurosurgery, Selcuk University, F }\end{array}$ & $\begin{array}{l}\text { niversity, Faculty of Medicine, Konya, Turkey } \\
\text { imleri University, Hamidiye, Turkey - Faculty of Medicine, Ministry of } \\
\text { of Medicine, Konya, Turkey } \\
\text { dicine, Konya, Turkey }\end{array}$ \\
\hline $\begin{array}{l}\text { Article } \\
\text { Information }\end{array}$ & $\begin{array}{l}\text { Article Type: Original Articles } \\
\text { Article Group: Pediatric Hematology-Oncology }\end{array}$ & $\begin{array}{l}\text { Received: } 16.11 .2020 \\
\text { Accepted: } 08.12 .2020 \\
\text { Available Online: } 27.12 .2020\end{array}$ \\
\hline
\end{tabular}

\section{Abstract}

Medulloblastoma is the most common central nervous system tumor in childhood. This study aims to evaluate the clinical features, treatments, and outcomes of pediatric patients diagnosed with medulloblastomaBetween 2006 and 2019, the medical records of children with medulloblastoma were reviewed retrospectively. Patients who died after surgery, before chemotherapy or radiotherapy were not included in the survival analysisDuring the study period, 38 children were diagnosed with medulloblastoma. Twenty-one of the patients were male (55.3\%) and 17 were female (44.7\%). The ages of the children ranged from 3 months to 17 years (median age 8 years). The ages of five patients were under 3 years (13.1\%). The most common complaints were headache ( $\mathrm{n}: 26,68.4 \%)$, imbalance ( $n: 21,55.3 \%$ ), and vomiting (n: 20, 52.6\%). The mass sizes ranged between 3 and $6 \mathrm{~cm}$ in 32 patients (84.2\%). At the time of diagnosis, 5 patients had seeding metastasis (13.1\%). The most commonlyused chemotherapy protocol included vincristine, cisplatin, etoposide (60.5\%). Five patients died after surgery without any chemotherapy or radiotherapy. Of the 33 patients included in the life analysis, 12 died (36.4\%). Follow-up times ranged from 2 months to 14 years (median, 44 months). The overall survival rate was $59.1 \%$. Eight patients had relapsed (24.2\%). Late relapse was detected in 3 of the relapsed patients (relapse times were the $91^{\text {st }}, 69^{\text {th }}$, and $72^{\text {nd }}$ months). It is possible to achieve satisfactory treatment results in children with medulloblastoma using international treatment guidelines and recommendations, with an experienced professional team dedicated to pediatric neurooncology.

Keywords: Children, medulloblastoma, treatment

Correspondence: Buket Kara, Department of Pediatric Hematology and Oncology, Selcuk University, Faculty of Medicine, Konya, Turkey E-mail: buketkara1001@gmail.com 


\section{Introduction}

Medulloblastoma generally locates in the posterior fossa, is the most common central nervous system (CNS) tumor in childhood, nearly $20 \%$ of all childhood CNS tumors. ${ }^{1,2}$ Generally, the metastasis is seen as seeding to the subarachnoid space, extra-neural metastasis can rarely be seen. ${ }^{3,4}$ The morphological variants of medulloblastoma are classic, large cell/ anaplastic, desmoplastic/ nodular medulloblastomas, and medulloblastoma with extensive nodularity. Together with molecular genetic studies, the World Health Organization made a new classification as Wingless (WNT)-activated, Sonic hedgehog (SHH)activated, Group 3, and Group 4 in medulloblastoma in $2016 .{ }^{2}$

Highlights
- Medulloblastoma is the most common
CNS tumor in children.
- The overall survival rate of the included
children with medulloblastoma was
$59.1 \%$.
- The event-free survival was $40.6 \%$.
- Satisfactory resultsof medulloblastoma
require an experienced neuro-oncology
team.

The treatment approach in patients with medulloblastoma is surgery, radiotherapy, and chemotherapy, respectively. In recent years, the improvement in the overall survival rates with the use of chemotherapy as well as surgery and radiotherapy in medulloblastoma has been detected. Also, the risk categories in medulloblastoma have been defined recently and the researchers are trying to plan the treatment approaches according to these risk categories. ${ }^{5}$ Survivals for patients with averageand high-risk were reported to be $82 \%$ and $45-50 \%$, respectively. ${ }^{1}$ In a large series from Hacettepe University, the overall survival rate was $43.1 \%$ in the whole group. ${ }^{6}$

In this study, from 2006 to 2019, the clinical features, treatments, and outcomes of pediatric patients diagnosed with medulloblastoma were evaluated retrospectively.

\section{Material and Method}

Between 2006 and 2019, the oncology charts of patients diagnosed, treated, and followed up with the diagnosis of medulloblastoma in our center were retrospectively reviewed. This study was approved by the Local Ethics Committee of Selçuk University (date: 17.12.2020, number: 2020/540). Demographic characteristics, symptoms and signs, radiologic and surgical findings, treatment approaches, and treatment outcomes were noted from the patients' oncologic charts.

The ages of the patients were grouped as $0-5$ years, $5-10$ years, and $>10$ years.

All patients' complaints have been noted and physical and neurological examinations have been carefully recorded. The primary tumor and spinal extension have been evaluated by craniospinal magnetic resonance imaging (MRI). Tumor size on MRI was divided into three groups as $<3 \mathrm{~cm}, 3-6 \mathrm{~cm}$, and $>6 \mathrm{~cm}$. Also, the presence of spinal seeding was investigated by MRI. In cases in which spinal seeding is suspected, a cerebrospinal fluid cytological examination was performed.
According to the surgical findings, they were classified as (i) total resection, (ii) subtotal resection, and (iii) only biopsy.

After surgery, all patients were treated with craniospinal radiotherapy (except the patients under age 3 years) and chemotherapy. The chemotherapy protocols used in order of frequency are

i. Cisplatin $\left(100 \mathrm{mg} / \mathrm{m}^{2} /\right.$ day, day 1 or $20 \mathrm{mg} / \mathrm{m}^{2} /$ day, days $1-5)$, etoposide $(100 \mathrm{mg} /$ $\mathrm{m}^{2}$ /day, days $\left.1-3\right)$, vincristine $\left(1.5 \mathrm{mg} / \mathrm{m}^{2} /\right.$ day, day 1$)$ with a 4-week interval thereafter;

ii. On cycles 1, 4, and 7: cisplatin $\left(20 \mathrm{mg} / \mathrm{m}^{2} /\right.$ day, days $1-5)$, etoposide $\left(100 \mathrm{mg} / \mathrm{m}^{2} /\right.$ day, days 1-3);

On cycles 2, 5, and 8: vincristine $\left(1.5 \mathrm{mg} / \mathrm{m}^{2} /\right.$ day, day 1), cyclophosphamide $\left(900 \mathrm{mg} / \mathrm{m}^{2} /\right.$ day, days 1 and 2$)$

On cycles 3, 6, and 9: Carboplatin $\left(150 \mathrm{mg} / \mathrm{m}^{2} /\right.$ day, days 1 and 15$)$, vincristine $\left(1.5 \mathrm{mg} / \mathrm{m}^{2} /\right.$ day, days 1 and 15).

iii. Chloroethylnitrosurea [(CCNU), $100 \mathrm{mg} /$ $\mathrm{m}^{2} /$ day, day 1], procarbazine $\left(100 \mathrm{mg} / \mathrm{m}^{2} /\right.$ day, days $1-14)$, vincristine $\left(1 \mathrm{mg} / \mathrm{m}^{2} /\right.$ day, days 1,8 , and 15$)$, prednisolone $\left(40 \mathrm{mg} / \mathrm{m}^{2} /\right.$ day, days $1-42$ days, only the first cycle) with 6-week intervals

The patients who died early after surgery before chemotherapy or radiotherapy were excluded from the survival analysis.

\section{Statistical analysis}

SPSS-15 software (SPSS Inc., Chicago, Illinois, USA) was used for all statistical analyses. Frequency and percentage values were used for categorical data, and minimum and maximum values were used in addition to the median value for numerical data.

Kaplan-Meier survival analysis was used for survival analyses. The patient groups were compared in terms of survival duration using a log-rank test. Alpha value (p) $<.05$ was considered significant.

\section{Results}

Between 2006 and 2019, 38 pediatric patients were diagnosed with medulloblastoma. The patients' demographic and clinical features are in Table 1. Twenty-one of the patients were male $(55.3 \%)$ and 17 were female $(44.7 \%)$. The patients' ages ranged from 3 months to 17 years (median age 8 years). Five patients were under 3 years $(13.1 \%)$.

The most common complaints were headache $(\mathrm{n}$ : $26,68.4 \%)$, imbalance (n: $21,55.3 \%$ ), and vomiting (n: $20,52.6 \%)$. The patients' mass sizes varied between 3 and $6 \mathrm{~cm}$ in 32 patients (84.2\%). Seeding metastasis was present in 5 patients at the time of diagnosis $(13.1 \%)$. 


\section{Table 1.}

The patients' demographic and clinical features

\section{n (\%)}

\begin{tabular}{lc}
\hline Age, median (minimum-maximum) & 8 years (3 months -17 years) \\
Age group & \\
$0-5$ years & $11,(28.9 \%)$ \\
$5-10$ years & $15,(39.5 \%)$ \\
$>10$ years & $12,(31.6 \%)$ \\
Gender & \\
Male & $21,(55.3 \%)$ \\
Female & $17,(44.7 \%)$ \\
Symptoms and signs & \\
Headache & $26,(68.4 \%)$ \\
Vomiting & $20,(52.6 \%)$ \\
Disturbances of gait and balance & $17,(44.7 \%)$ \\
Strabismus & $5,(13.2 \%)$ \\
Diplopia & $5,(13.2 \%)$ \\
Head tilt & $5,(13.2 \%)$ \\
Mental disturbances & $1,(2.6 \%)$ \\
Mass size & \\
<3 cm & $3,(7.9 \%)$ \\
cm & $32,(84.2 \%)$ \\
>6 cm & $3,(7.9 \%)$ \\
Seeding & $5,(13.2 \%)$ \\
Surgery & \\
Total & $31,(81.5 \%)$ \\
Subtotal & $5,(13.2 \%)$ \\
Only biopsy & $2,(5.3 \%)$ \\
Pathology & \\
Classic & $35,(92.1)$ \\
Nodular & $2,(5.3 \%)$ \\
Desmoplastic & $1,(2,6 \%)$ \\
\hline &
\end{tabular}

The most commonly used chemotherapy protocol included vincristine, cisplatin, etoposide (60.5\%). Five patients died without any treatment (chemotherapy or radiotherapy) in the early period after surgery.

Twelve of the 33 patients included in the life analysis died (36.4\%). Follow-up times ranged from 2 months to 14 years (median, 44 months). The overall survival (OS) and event-free survival (EFS) rates were $59.1 \%$ and $40.6 \%$, respectively (Figure 1).

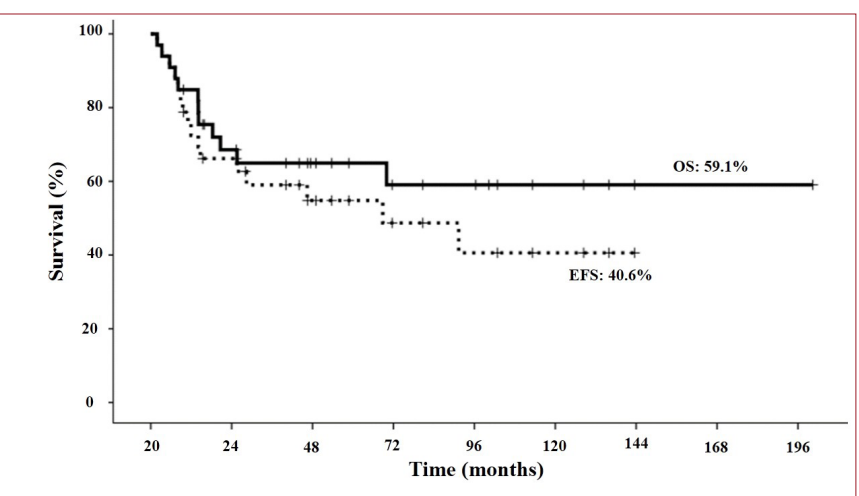

Figure 1. The rates of overall survival and event free survival of all patients

Eight patients had arelapse (24.2\%). Late relapses were detected in 3 of the patients (relapse times were the $91^{\text {st }}$, $69^{\text {th }}$, and $72^{\text {nd }}$ months).

\section{Discusion}

Medulloblastoma is the most common CNS tumor in childhood and constitutes approximately $20 \%$ of all CNS tumors in children aged 0-14 years. Although they are usually diagnosed in the first decade of life, it can be diagnosed in older children and even in adult age groups. It is more common in males than females. Although its etiology is not known exactly, its association with some inherited syndromes such as Gorlin-Goltz syndrome, Turcot syndrome, LiFraumeni syndrome, neurofibromatosis type 1 and 2, Rubinstein-Taybi syndromes, Fanconi anemia, and Nijmegen breakage syndrome have been reported. ${ }^{1,2}$

In this study, the clinical features, treatments and treatment outcomes of pediatric patients diagnosed with medulloblastoma were evaluated retrospectively.

Although the age of our patients ranged from three months to 17 years (median, 8 years), approximately two-thirds of the patients were under 10 years old. There was a slight male dominance in gender distribution. These demographic features were similar to the literature.

Medulloblastoma is generally located posterior fossa and associated with the fourth ventricle. Clinical findings include headache, vomiting, papillary edema, irritability, diplopia, nystagmus, and rapid growth of head circumference during infancy. These findings occur due to hydrocephalus and increased intracranial pressure secondary to tumor obstruction. The most common symptoms are vomiting and headache and they are present in $80 \%$ of patients. ${ }^{1,2}$ The most common complaints in our patients were headache, vomiting, and disturbances of gait and balance. Other findings such as strabismus, diplopia, head tilt, and mental disturbances were less frequent. ${ }^{?}$

In medulloblastoma, theinitial treatment approach is surgery. The main purpose of surgery is total or neartotal resection of the tumor and, if possible, remaining residual tumor less than $1.5 \mathrm{~cm}^{2}$, and this is one of the main prognostic factors. ${ }^{1,2,5,6}$ In our study, it was learned from the patients' charts that a significant portion of our patients underwent total or near-total resection. However, because our study was retrospective and some patients were referred from another center, we were unfortunately not informed about the residual tumor volume due to the lack of early radiological examination. This was one of the limitations of our study.

Anothertreatmentthathasacritical roleinmedulloblastoma is radiotherapy.Radiotherapy application is craniospinal radiotherapy. However, an important limitation in radiotherapy is radiotherapy-related side effects such as neurocognitive, endocrinological and growth side effects in children under three years of age. . $^{1,2,5,6} \mathrm{In}$ our patients, radiotherapy could not be applied in five patients younger than three years old. The others received appropriate radiotherapy for the risk group.

Chemotherapy is currently considered as a standard adjuvant treatment modality. Many chemotherapeutic agents, vincristine, cisplatin, etoposide and alkylating agents are used. ${ }^{1,2,5,6}$ Cisplatin-based chemotherapy 

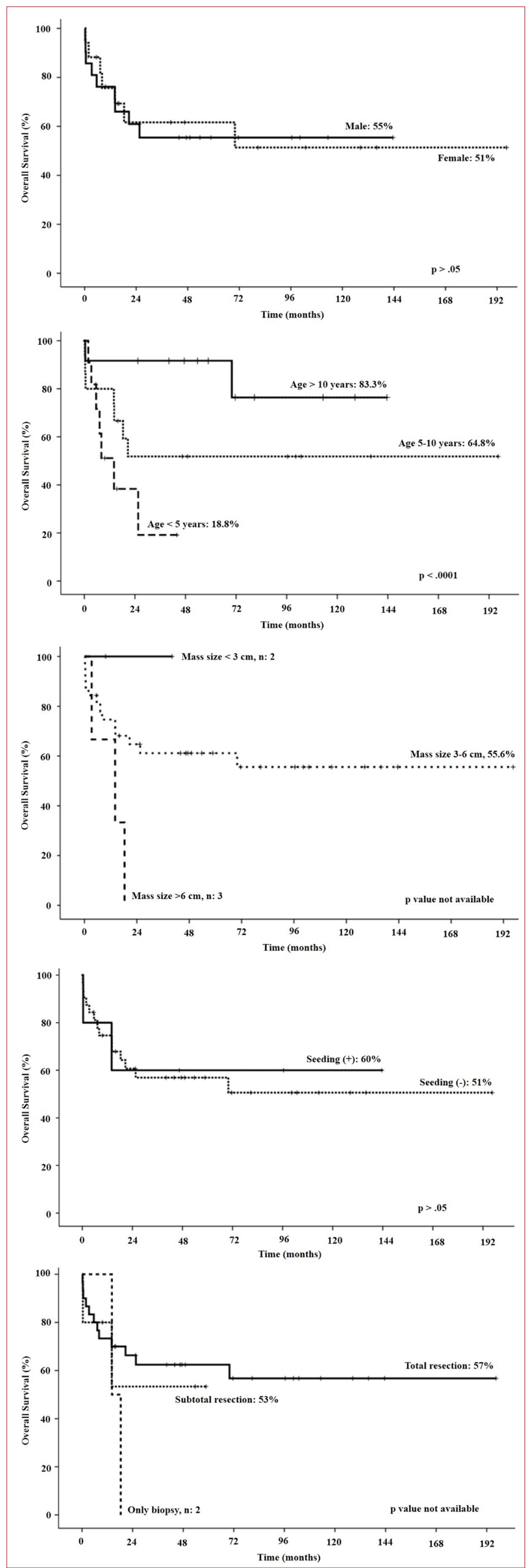

Figure 2. The factors affecting overall survival protocols were mostly used in our patients. While the first applied chemotherapy scheme was vincristine, cisplatin, etoposide, alternating with cisplatin, etoposide (on the 1, 4 , and 7. cycles), vincristine, cyclophosphamide (on the 2,5 , and 8 . cycles), carboplatin, vincristine (on the 3,6 , and 9 . cycles) chemotherapy schemes were used in the following years.

Medulloblastoma is divided into two as standard and high risk according to its prognostic factors. The standard risk group is $>3$ years old, no metastatic disease at diagnosis, tumor resected totally or nearly total, residual tumor size $<1.5 \mathrm{~cm}^{2}$. High-risk group is being younger than 3 years old, the presence of metastases at diagnosis, or determination of $>1.5 \mathrm{~cm}^{2}$ tumor mass after resection.Medulloblastoma has four molecular subgroups as WNT tumors, Sonic Hedgehog tumors, Group 3, and Group 4 tumors. Groups 3, and 4 medulloblastomas are considered as non-WNT, non-Sonic Hedgehog tumors. Retrospective studies have shown that this classification has important prognostic significance. While survival rates in patients with medulloblastoma in which the WNT pathway is activated are determined to be above $90 \%$, Sonic Hedgehog tumors subtype and Group 4 tumors have a moderate survival rate (75\%). Group 3 cases have the worst survival rates of $40-60 \% .^{7}$ Another limitation of our study is that molecular subgroups could not be determined, especially since it was a retrospective study.

For standard and high-risk medulloblastoma, the overall survival rates are nearly $80 \%$ and $50 \%$, respectively. ${ }^{1}$ The overall survival rate was reported as $43.1 \%$ in the whole group from a big center. ${ }^{6}$ In our study, the OS and EFS rates were $59.1 \%$ and $40.6 \%$, respectively. It may contribute more positively to survival rates by determining a more reliable risk group and molecular subgroups.

\section{Conclusion}

In medulloblastoma, which is the most common CNS tumor of childhood, multidisciplinary approaches both at the diagnosis and treatment will have positive contributions to the treatment success of the disease

Acknowledgement: We would like to thank all patients and families for their participation in this study.

Author Contributions: YK conceived the study. YK and BK were involved in patient care, including the process of procedure and routine clinical follow-up. YK, BK, HA and MD performed the literature review and wrote the manuscript. YK also made statistical analysis. HA, MD and $\mathrm{HK}$ also made helpful suggestions to improve the manuscript.

Conflict of Interest Statement: The authors declared no potential conflicts of interest with respect to the research, authorship,and/or publication of this article.

Ethics Committee Approval: This study was approved by the Local Ethics Committee of Selçuk University (date: 17.12.2020, number: 2020/540). 
Financial Disclosure: The authors declared that this study has received no financial support.

Informed Consent: Because the study was designed retrospectively, no written informed consent form was obtained from patients.

Peer-review: Externally peer-reviewed.

\section{References}

1. Hanson DR, Atlas MP. Central nervous system malignancies. In Lanskowsky P, Lipton JM, Fish JD, eds. Lanskowsky's Manual of Pediatric Hematology and Oncology, 6th ed. Amsterdam: Elsevier, 2016: 453-472.

2. Cassia GSE, Alves CAPF, Taranath A, et al. Childhood Medulloblastoma Revisited. Top Magn Reson Imaging. 2018;27:479-502. [CrossRef]
3. Akyüz C, Yalçin B, Kutluk T, Cila A, Büyükpamukçu M. Brief report: Medulloblastoma with widespread skeletal metastases presenting with hypercalcemia. Med Pediatr Oncol. 1999;33:126128.[CrossRef]

4. Varan A, Sari N, Akalan N, et al. Extraneural metastasis in intracranial tumors in children: the experience of a single center. $J$ Neurooncol. 2006;79:187-190. [CrossRef]

5. Varan A. Risk-adapted chemotherapy in childhood medulloblastoma. Expert Rev Anticancer Ther. 2011;11:771-780. [CrossRef]

6. Akyüz C, Varan A, Küpeli $S$, et al. Medulloblastoma in children: a 32-year experience from a single institution. J Neurooncol. 2008;90:99-103. [CrossRef]

7. Tumturk A, Kaya Ozcora G, Kacar Bayram A, et al. Torticollis in children: an alert symptom not to be turned away. Childs Nerv Syst. 2015;31:1461-1470.

8. Ertan Y, Sezak M, Demirağ B, et al. Medulloblastoma: clinicopathologic evaluation of 42 pediatric cases. Childs Nerv Syst. 2009;25:353-356. [CrossRef] 